\title{
ÍNDICE ANALÍTICO
}

Acción de apoyo civil y militar de la Unión Europea a la misión de la Unión Africana en la región sudanesa de Darfur, UE-2755

Acciones del séptimo programa marco de la Comunidad Europea de la Energía Atómica, y las normas de difusión de los resultados de la investigación, UE-2647

Acogida temporal por los Estados miembros de la Unión Europea de determinados palestinos, UE-2769

Acta de adhesión de Bulgaria a la Unión Europea, UE-2637

Acta de adhesión de la República de Bulgaria y Rumanía en lo referente al desarrollo rural, UE-2535

Acta de Ginebra del Arreglo de La Haya relativo al Registro internacional de dibujos y modelos industriales, UE-2629, UE-2738, UE-2739

Acuerdo CE-República de Belarús sobre comercio de productos textiles, UE-2465, UE-2628

Acuerdo de Asociación en el sector pesquero entre la Comunidad Europea y la República Islámica de Mauritania, UE-2543, UE-2626

Acuerdo de asociación en el sector pesquero entre la Comunidad Europea y la República de Cabo Verde, UE-2545, UE-2633

Acuerdo de asociación en materia de pesca entre la Comunidad Europea, por una parte, y el Gobierno de Dinamarca y el Gobierno Autónomo de Groenlandia, por otra, UE-2544

Acuerdo de colaboración en el sector pesquero entre la Comunidad Europea y la República de Seychelles, UE-2540

Acuerdo de colaboración en el sector pesquero entre la Comunidad Europea y la Unión de las Comoras, UE-2541

Acuerdo de Cooperación sobre un sistema mundial civil de navegación por satélite (GNSS) entre la Comunidad Europea y sus Estados miembros, por una parte, y la República de Corea, por otra, UE-2724

Acuerdo de estabilización y asociación entre las Comunidades Europeas y sus Estados miembros, por una parte, y la República de Albania, UE-2624 Acuerdo en forma de Canje de Notas entre la Unión Europea y el Gobierno de Indonesia sobre los cometidos, el estatuto, los privilegios y las inmunidades de la Misión de Observación de la Unión Europea en Aceh, UE-2747 Acuerdo en forma de Canje de Notas entre la Unión Europea y el Gobierno de la Confederación Suiza sobre la participación de la Confederación Suiza en la Operación Militar de la Unión Europea de apoyo a la Misión de las 
Naciones Unidas en la República Democrática del Congo (MONUC) durante el proceso electoral, UE-2748

Acuerdo entre el Consejo de ministros de la República de Albania y la Comunidad Europea sobre determinados aspectos de los servicios aéreos, UE-2568

Acuerdo entre la Comunidad Europea y el Gobierno de Canadá por el que se instituye un marco de cooperación en materia de enseñanza superior, formación y juventud, UE-2632, UE-2716

Acuerdo entre la Comunidad Europea y el Gobierno de la República de Guinea-Bissau relativo a la pesca, UE-2539, UE-2623

Acuerdo entre la Comunidad Europea y el Gobierno de Malasia sobre determinados aspectos de los servicios aéreos, UE-2574, UE-2634

Acuerdo entre la Comunidad Europea y el Reino de Marruecos sobre determinados aspectos de los servicios aéreos, UE-2572, UE-2630

Acuerdo entre la Comunidad Europea y la República de Chile sobre determinados aspectos de los servicios aéreos, UE-2569

Acuerdo entre la Comunidad Europea y la República de Chile sobre determinados aspectos de los servicios aéreos, UE-2570

Acuerdo entre la Comunidad Europea y la República de Maldivas sobre determinados aspectos de los servicios aéreos, UE-2567

Acuerdo entre la Comunidad Europea y la República Gabonesa sobre la pesca, UE-2542, UE-2625

Acuerdo entre la Comunidad Europea y la República Oriental del Uruguay sobre determinados aspectos de los servicios aéreos, UE-2571

Acuerdo entre la Unión Europea y la República de Islandia y el Reino de Noruega sobre el procedimiento de entrega entre los Estados miembros de la Unión Europea e Islandia y Noruega, UE-2774

Acuerdo entre la Unión Europea y los Estados Unidos de América sobre el tratamiento y la transferencia de datos del registro de nombres de los pasajeros (PNR) por las compañías aéreas al Departamento de Seguridad del Territorio Nacional de los Estados Unidos, UE-2749, UE-2776

Acuerdo Euromediterráneo de Aviación entre la Comunidad Europea y sus Estados miembros, por una parte, y el Reino de Marruecos, por otra, UE-2573, UE-2631

Acuerdo Gobierno EE.UU-CE sobre la coordinación de los programas de etiquetado de la eficiencia energética para los equipos ofimáticos, UE-2464, UE-2627

Acuerdo interino entre la Comunidad Europea y la República de Albania, UE-2624

Acuerdo marco sobre un Programa Multilateral de Medio Ambiente en el Ámbito Nuclear en la Federación Rusa, UE-2645 
Acuerdo Multilateral entre la Comunidad Europea y sus Estados miembros, la República de Albania, la Antigua República Yugoslava de Macedonia, Bosnia y Herzegovina, la República de Bulgaria, la República de Croacia, la República de Islandia, la República de Montenegro, el Reino de Noruega, Rumanía, la República de Serbia y la Misión de Administración Provisional de las Naciones Unidas en Kosovo, sobre la creación de una Zona Europea Común de Aviación (ZECA), UE-2566

Acuerdo sobre la conservación de las aves acuáticas migratorias afroeurasiáticas, UE-2691

Acuerdo sobre la constitución de la Organización Internacional de la Energía de Fusión ITER para la Ejecución Conjunta del Proyecto ITER, UE-2646

Acuerdo sobre los privilegios e inmunidades de la Organización Internacional de la Energía de Fusión ITER para la Ejecución Conjunta del Proyecto ITER, UE-2646

Adición de vitaminas, minerales y otras sustancias determinadas a los alimentos, UE-2667

Administración Autonómica: GAL-18

Adopción Internacional: EST-95

Agencia Europea de Reconstrucción, UE-2635

Agencia Europea de Seguridad Marítima, UE-2583

Agencia Europea de Sustancias y Preparados Químicos, UE-2665, UE-2679

Agencia Tributaria de Cataluña: CAT-2

Alimentos elaborados a base de cereales y alimentos infantiles para lactantes y niños de corta edad, UE-2671

Año Europeo del Diálogo Intercultural (2008), UE-2723

Aplicación de las reservas mínimas por el Banco Central Europeo después de la introducción del euro en Eslovenia, UE-2613

Aplicación de los artículos 87 y 88 del Tratado a las ayudas de minimis, UE-2603 Aplicación de los artículos 87 y 88 del Tratado a las ayudas estatales para las pequeñas y medianas empresas dedicadas a la producción de productos agrícolas, UE-2600

Aplicación de los artículos 87 y 88 del Tratado a las ayudas regionales a la inversión, UE-2598

Aplicación de los regímenes de seguridad social a los trabajadores por cuenta ajena, a los trabajadores por cuenta propia y a sus familias que se desplazan dentro de la Comunidad, UE-2561

Aplicación del artículo 36 del Tratado Euratom sobre el control de los índices de radiactividad en el medio ambiente, UE-2653

Aplicación del artículo 81, apartado 3, del Tratado a determinadas categorías de acuerdos y prácticas concertadas, UE-2595

Aplicación del régimen agromonetario del euro en el sector agrario, UE-2515 
Aproximación de las legislaciones de los Estados miembros, UE-2584, UE2585, UE-2674, UE-2675, UE-2679

Aranceles aduaneros sobre la glucosa y la lactosa, UE-2458

Armonización de normas técnicas y procedimientos administrativos aplicables a la aviación civil, UE-2581, UE-2582

Armonización del espectro radioeléctrico para los dispositivos de identificación por radiofrecuencia (RFID) que utilizan la banda de frecuencia ultraalta (UHF), UE-2682

Armonización del espectro radioeléctrico para su uso por dispositivos de corto alcance, UE-2690

Artesanía AND-40

Asociaciones: PV-4

Auditor externo del Banco de España, UE-2620

Autorización de las ayudas estatales en el marco de las disposiciones de los artículos 87 y 88 del Tratado CE, UE-2605, UE-2606, UE-2607

Autorización permanente de un aditivo en la alimentación animal, UE-2503

Ayuda financiera de la Comunidad a los laboratorios comunitarios de referencia para los piensos, los alimentos y el sector de la salud animal, UE-2506

Ayuda financiera excepcional de la Comunidad a Kosovo, UE-2758

Ayuda para los gusanos de seda, UE-2504

Ayudas otorgadas por los Estados miembros a las organizaciones de productores del sector de la pesca y la acuicultura, UE-2557

Banco de Tierras de Galicia: GAL-19

Base de Datos Policial: EST-89

Biodiversidad: EST-100

Buenas prácticas de fabricación de materiales y objetos destinados a entrar en contacto con alimentos, UE-2664

Buques que practiquen la pesca ilegal, no declarada y no reglamentada en el Atlántico Nordeste, UE-2548

Calidad Agroalimentaria: AND-41

Calidad del Aire: EST-98

Calidad exigida a las aguas para cría de moluscos, UE-2696

Capital europea de la cultura para el año 2010, UE-2720

Capital Europea de la Cultura para los años 2007 a 2019, UE-2719

Caracteres que los exámenes deben analizar como mínimo y las condiciones mínimas para examinar determinadas variedades de especies de plantas hortícolas, UE-2533

Carrera Militar: EST-64

Carta Europea de Calidad para la Movilidad, UE-2714 
Castilla y Leon: EST-62

Caza: AND-42

Centro Catalán de Estudios de Opinión: CAT-1.

Centro de Atención y Gestión de Llamadas de Urgencia 112: CAT-10

Centro de satélites de la Unión Europea, UE-2766

Centro Técnico de Cooperación Agrícola y Rural (CTA), UE-2644

Certificación del lúpulo y productos del lúpulo, UE-2511

Cine: EST-63

Clasificación, embalaje y etiquetado de las sustancias peligrosas, UE-2679

Codificación, procesamiento, preservación, almacenamiento y distribución de células y tejidos humanos, UE-2694

Código aduanero comunitario, UE-2459

Código y normas tipo relativas a la transcripción en forma legible por máquina de las encuestas de base sobre las superficies vitícolas, UE-2537

Combatir la acumulación y la proliferación desestabilizadoras de armas ligeras

y de pequeño calibre en América Latina y el Caribe, UE-2759

Comercialización de plantones de hortalizas, UE-2532

Comercialización de preparados para lactantes a base de hidrolizados de proteínas de lactosuero, UE-2491

Comercialización de semillas de plantas hortícolas, UE-2532

Comercialización y uso de determinadas sustancias y preparados peligrosos, UE-2674

Comercialización y uso de los compuestos de arsénico, UE-2678

Comercio de determinados productos siderúrgicos CE-Federación de Rusia, UE-2471

Comercio de determinados productos siderúrgicos CE-República de Kazan, UE-2469

Comercio de determinados productos siderúrgicos CE-Ucrania, UE-2470

Comisión Nacional del Sector Público: EST-105

Comité de estadísticas monetarias, financieras y de balanza de pagos, UE-2617

Competencias clave para el aprendizaje permanente, UE-2715

Comunicaciones Electrónicas: EST-73

Concierto Económico con la Comunidad Autónoma del Pais Vasco: EST-84

Condiciones de acceso a la red para los intercambios transfronterizos de electricidad, UE-2689

Condiciones de importación de productos agrícolas originarios de terceros países como consecuencia del accidente ocurrido en la central nuclear de Chernobil, UE-2493

Conferencia de La Haya de Derecho Internacional Privado, UE-2775

Consejo de Relaciones Laborales: CAT-8

Consejo de Seguridad Nuclear: EST-80 
Contenido de la información contable que deberá presentarse a la Comisión con vistas a la liquidación de cuentas del FEAGA y del FEADER, UE-2479

Contenido máximo de determinados contaminantes en los productos alimenticios, UE-2660

Contingentes arancelarios comunitarios autónomos para determinados productos agrícolas e industriales, UE-2460

Contingentes arancelarios comunitarios para la importación en el sector de los cereales, UE-2461

Contratos del Sector Público: EST-82

Control de conformidad con las normas de comercialización aplicables a las frutas y hortalizas frescas efectuadas en Turquía antes de la importación en la Comunidad Europea, UE-2507

Convenio de Rotterdam sobre el procedimiento de consentimiento fundamentado previo aplicable a ciertos plaguicidas y productos químicos peligrosos objeto de comercio internacional, UE-2463

Convenio de Schengen, modificación, UE-2781

Convenio Económico entre el Estado y la Comunidad Foral de Navarra: EST-87

Convenio relativo a la simplificación de formalidades en los intercambios de mercancías, UE-2462

Criterios específicos de pureza de los aditivos alimentarios distintos de los colorantes y edulcorantes, UE-2673

Criterios específicos de pureza de los edulcorantes que pueden emplearse en los productos alimenticios, UE-2672

Cuentas anuales del Banco Central Europeo, UE-2618

Cuidado de los Animales: EST-61

Cupo del Pais Vasco: EST-85

Declaración de la Presidencia del Consejo de la Unión Europea y de la Comisión Europea sobre la República de Guinea, UE-2773

Declaraciones nutricionales y de propiedades saludables en los alimentos, UE-2666

Derecho antidumping definitivo, UE-2596, UE-2597, UE-2599, UE-2601, UE-2602

Derecho Civil: GAL-20

Derechos de alquiler y préstamo y otros derechos afines a los derechos de autor en el ámbito de la propiedad intelectual, UE-2742

Derechos sociales: PV-5

Destilación de crisis para determinados vinos de España, UE-2485

Diario Oficial de la Generalitat de Cataluña: CAT-4

Directrices estratégicas comunitarias en materia de cohesión, UE-2686 
Disposiciones de aplicación del artículo 93 del Tratado CE, UE-2608

El Defensor del Pueblo Europeo: Informe Anual del año 2005, UE-2743

Empresa Común Galileo, UE-2725

Empresas de Inserción: EST-77

Energía: AND-46

Enseñanzas no universitarias: AND-44; AND-45

Equipo de planificación de la UE (EPUE Kosovo), UE-2765

Especificación técnica de interoperabilidad referente al subsistema de control y mando y señalización del sistema ferroviario transeuropeo convencional, UE-2591

Especificación técnica de interoperabilidad referente al subsistema material rodante-vagones de mercancías del sistema ferroviario transeuropeo convencional, UE-2593

Especificación técnica de interoperabilidad referente al subsistema Explotación y gestión del tráfico del sistema ferroviario transeuropeo convencional, UE-2594

Especificación técnica de interoperabilidad relativa al subsistema controlmando y señalización del sistema ferroviario transeuropeo de alta velocidad, UE-2592

Espectáculos públicos: AND-47

Establecimientos comerciales: AND-48

Estadísticas coyunturales, en lo que respecta a las excepciones concedidas a los Estados miembros, UE-2708

Estadísticas coyunturales, en lo relativo a la definición de las variables, la lista de variables y la frecuencia de compilación de datos, UE-2709

Estadísticas del comercio exterior de la Comunidad y del comercio entre sus Estados miembros, UE-2468

Estatuto de los Trabajadores: EST-102

Etiqueta ecológica comunitaria a las enmiendas del suelo, UE-2699

Explotaciones ganaderas: AND-49

\section{Farmacias: AST-6}

Ficha de explotación que debe utilizarse para el registro de las rentas de las explotaciones agrícolas, UE-2513

Financiación de la normalización europea, UE-2681

Flotas pesqueras registradas en las regiones ultraperiféricas de la Comunidad, UE-2550

Fondo de Cohesión, UE-2684

Fondo Europeo Agrícola de Desarrollo Rural (FEADER), UE-2482, UE-2517, UE-2519, UE-2524 
Fondo Europeo Agrícola de Garantía (FEAGA), UE-2523

Fondo Europeo de Adaptación a la Globalización, UE-2685

Fondo Europeo de Desarrollo Regional, UE-2684, UE-2687

Fondo Europeo de Orientación y de Garantía Agrícola (FEOGA), UE-2536

Fondo Social Europeo, UE-2684

Forma de evitar el doble cómputo de las reducciones de emisiones de gases de efecto invernadero en el régimen comunitario, UE-2697

Franquicias aplicables a la importación de mercancías, UE-2609

Función pública: GAL-21

Garantía de la Comunidad al Banco Europeo de Inversiones, UE-2640

Gestión Integrada de la Calidad Ambiental: AND-50

Guardia Civil: EST-90, EST-91, EST-92

Higiene de los productos alimenticios y a las normas sanitarias que regulan la producción y comercialización de determinados productos de origen animal destinados al consumo humano, UE-2707

Homologación de los vehículos de motor de dos o tres ruedas, UE-2670

Identificación electrónica de los animales de las especies ovina y caprina, UE-2538

Identificación y el registro de los animales de las especies ovina y caprina, UE-2483

Impuesto sobre la Renta de las Personas Físicas: EST-86

Información contenida en los folletos así como al formato, la incorporación por referencia, la publicación de dichos folletos y la difusión de publicidad, UE-2565, UE-2658

Información del Sector Público: EST-83

Información sobre los ordenantes que acompaña a las transferencias de fondos, UE-2659

Inicio de procedimientos de solución de diferencias contra la India, UE-2474

Inmigración clandestina: EST-94

Instituto Andaluz del Patrimonio Histórico: AND-52

Instituto Catalán de la Salud: CAT-9

Instituto de Estudios de Seguridad de la Unión Europea, UE-2767

Instituto de Seguridad Pública de Cataluña: CAT-3

Instituto Europeo de la Igualdad de Género, UE-2562, UE-2563, UE-2744, UE-2745

Instituto Vasco de Consumo: PV-3

Instrumento de Estabilidad, UE-2636

Instrumento de Financiación de la Cooperación al Desarrollo, UE-2641 
Instrumento de financiación de la cooperación con los países y territorios industrializados y otros países y territorios de renta alta, UE-2643

Instrumento Europeo de Vecindad y Asociación, UE-2751

Instrumento financiero para la promoción de la democracia y de los derechos humanos a escala mundial, UE-2642

Instrumentos de medida, UE-2683

Introducción del euro, UE-2614

Irregularidades y a la recuperación de las sumas indebidamente pagadas en el marco de la financiación de la política agrícola común, UE-2510

Licitaciones permanentes para la reventa en el mercado comunitario de cereales, UE-2481

Licitaciones permanentes para la reventa, en el mercado comunitario, de trigo blando y centeno, UE-2480

Límites de captura de las poblaciones de espadín, UE-2549

Lista comunitaria de las compañías aéreas objeto de una prohibición de explotación en la Comunidad, UE-2575

Lista de organismos cuyos investigadores pueden acceder, con fines científicos, a datos confidenciales, UE-2712

Lista de terceros países cuyos nacionales están sometidos a la obligación de visado para cruzar las fronteras exteriores y la lista de terceros países cuyos nacionales están exentos de esa obligación, UE-2782

Lucha contra el piojo de San José, UE-2530, UE-2668

Mantequilla de intervención puesta a la venta, UE-2478, UE-2508

Manual Sirene, UE-2787, UE-2788

Material de Defensa: EST-65

Material eléctrico destinado a utilizarse con determinados límites de tensión, UE-2675

Mecanismo de cooperación y verificación de los avances logrados por Rumanía para cumplir indicadores concretos en materia de reforma judicial y lucha contra la corrupción, UE-2638

Mecanismo de cooperación y verificación de los avances logrados por Bulgaria para cumplir indicadores concretos en materia de reforma judicial, lucha contra la corrupción y la delincuencia organizada, UE-2639

Mecanismo de información mutua sobre las medidas de los Estados miembros en materia de asilo e inmigración, UE-2785

Mediación familiar: AST-7

Medicamentos para uso pediátrico, UE-2662, UE-2663

Medidas de aplicación de determinados productos de origen animal destinados al consumo humano, UE-2704 
Medidas de gestión para la explotación sostenible de los recursos pesqueros en el Mar Mediterráneo, UE-2560

Medidas de prevención y lucha contra la violencia y los desórdenes relacionados con los partidos de fútbol de dimensión internacional en los que se vea afectado al menos un Estado miembro, UE-2790

Medidas en apoyo de la aplicación efectiva del mandato del Tribunal Penal Internacional para la ex Yugoslavia (TPIY), UE-2768

Medidas especiales para favorecer la cría de gusanos de seda, UE-2486

Medidas específicas de lucha contra el terrorismo, UE-2772

Medidas fiscales y financieras: CAT-5

Medidas para proteger más eficazmente los billetes en euros contra la falsificación, UE-2619

Medidas restrictivas contra determinados funcionarios de Belarús, UE-2754

Medidas restrictivas contra el Presidente Lukashenko y determinados funcionarios de Belarús, UE-2750

Medidas restrictivas contra la República Popular Democrática de Corea, UE-2771

Medidas restrictivas contra Liberia, UE-2752

Medidas restrictivas contra Uzbekistán, UE-2770

Medidas restrictivas específicas dirigidas a determinadas personas y entidades con el fin de luchar contra el terrorismo, UE-2789

Medidas restrictivas específicas dirigidas contra determinadas personas y entidades asociadas con Usamah bin Ladin, la red Al-Qaida y los talibanes, UE-2753

Medio Rural: EST-99, EST-101

Mercado de valores: EST-78

Mercado hipotario: EST-75

Métodos alternativos de eliminación y utilización de subproductos animales, UE-2498

Métodos de muestreo y de análisis para el control oficial del contenido de nitratos en ciertos productos alimenticios, UE-2661

Ministerio Fiscal: EST-93

Misión de asistencia fronteriza de la Unión Europea para el paso fronterizo de Rafah (MAFUE Rafah), UE-2761

Misión de Observación de la Unión Europea (MOUE), UE-2762

Misión de Policía de la Unión Europea (MPUE) en Bosnia y Herzegovina, UE-2757

Misión de Policía de la Unión Europea en Kinshasa (RDC) relativa a la Unidad integrada de Policía (EUPOL Kinshasa), UE-2763, UE-2764

Misión de Policía de la Unión Europea para los Territorios Palestinos, UE-2756 
Misión Integrada de la Unión Europea por el Estado de Derecho en Iraq, EUJUST LEX, UE-2760

Niveles de emisión asignados a la Comunidad y a cada uno de sus Estados miembros con arreglo al Protocolo de Kyoto, UE-2700

Nomenclatura estadística de actividades económicas NACE Revisión 2, UE-2710

Normas comunes de seguridad aérea, UE-2576, UE-2579

Normas comunes para determinados transportes de mercancías por carretera, UE-2587

Normas específicas de higiene de los alimentos de origen animal, UE-2702

Normas específicas para la organización de controles oficiales de los productos de origen animal destinados al consumo humano, UE-2703

Normas específicas para los controles oficiales de la presencia de triquinas en la carne, UE-2705

Normas relativas al tráfico fronterizo menor en las fronteras terrestres exteriores de los Estados miembros, UE-2781

Obras públicas: CAT-6

Observatorio Europeo de las Drogas y las Toxicomanías, UE-2706

Organización común de mercados en el sector de los productos de la pesca y de la acuicultura, UE-2551

Organización común del mercado vitivinícola, UE-2484, UE-2518

Partidos políticos: EST-69

Patrimonio: PV-7

Permiso de conducción, UE-2590

Perros de asistencia: PV-6

Persecución o violencia durante la guerra civil y la dictadura: EST-70

Personas con discapacidad: EST-68

Personad sordas: EST-67

Plazo de protección del derecho de autor y de determinados derechos afines,

\section{UE-2741}

Policía de Galicia GAL-22

Policía Sanitaria Mortuoria: AND-53

Policías locales: AST-8

Política monetaria del Eurosistema, UE-2622

Posibilidades de pesca de determinadas poblaciones de peces de aguas profundas, UE-2556

Posibilidades de pesca y las condiciones asociadas a determinadas poblaciones de peces, UE-2553 
Posibilidades de pesca y las condiciones asociadas aplicables en el Mar Báltico a determinadas poblaciones y grupos de poblaciones de peces, UE-2555 Preparados para lactantes y preparados de continuación, UE-2680

Prescripciones técnicas de las embarcaciones de la navegación interior, UE-2588, UE-2589

Presupuestos Generales del Estado: EST-88

Presupuestos: CAT-7; PV-8

Prevención y el control de determinadas enfermedades de los animales acuáticos, UE-2531

Prevención, el control y la erradicación de determinadas encefalopatías espongiformes transmisibles, UE-2528

Principio de reconocimiento mutuo de resoluciones de decomiso, UE-2783

Procedimientos de aceptación de los cereales por los organismos de intervención y los métodos de análisis para la determinación de la calidad, UE-2489 Procedimientos de consulta y de información en materia de seguros de crédito, garantías y créditos financieros, UE-2473

Procedimientos de contratación en los sectores del agua, la energia, los transportes y los servicios postales: EST-72

Procedimientos de control y la condicionalidad en relación con las medidas de ayuda al desarrollo rural, UE-2520

Proceso monitorio europeo, UE-2780

Producción agrícola ecológica y su indicación en los productos agrarios y alimenticios, UE-2521, UE-2529

Productos siderúrgicos originarios de determinados terceros países, UE-2472 Programa comunitario para el empleo y la solidaridad social - Progress, UE-2564 Programa Cultura, UE-2721, UE-2722

Programa de acción comunitaria en el ámbito de la política de los consumidores, UE-2701

Programa de acción en el ámbito del aprendizaje permanente, UE-2718

Programa de acción en materia de intercambios, asistencia y formación para la protección del euro contra la falsificación de moneda (programa Pericles), UE-2615, UE-2616

Programa de apoyo al sector audiovisual europeo (MEDIA 2007), UE-2713

Programa Europa con los ciudadanos, para promover la ciudadanía europea activa, UE-2746

Programa europeo de radionavegación por satélite, UE-2580

Programa La juventud en acción, UE-2717

Programa marco para la innovación y la competitividad, UE-2656, UE-2688, UE-2698

Prohibición de la pesca de brótola de fango por parte de los buques que enarbolan pabellón de España, UE-2546, UE-2547 
Prohibición la pesca de fletán negro por parte de los buques que enarbolan pabellón de España, UE-2554

Promoción y defensa de la competencia: AND-43

Protección de las aguas subterráneas contra la contaminación y el deterioro, UE-2695

Protección de las indicaciones geográficas y de las denominaciones de origen de los productos agrícolas y alimenticios, UE-2740

Protección de los Consumidores: EST-76

Protección de los menores y de la dignidad humana y al derecho de réplica en relación con la competitividad de la industria europea de servicios audiovisuales y de información en línea, UE-2657

Protocolo del Acuerdo Europeo por el que se crea una asociación entre las Comunidades Europeas y sus Estados miembros, por una parte, y Rumanía, por otra, sobre evaluación de la conformidad y aceptación de productos industriales (PECA), UE-2655

Publicidad engañosa y publicidad comparativa, UE-2676

Recursos financieros destinados a la clausura de instalaciones nucleares y a la gestión del combustible gastado y los residuos radiactivos, UE-2654

Reestructuración y reconversión de viñedos, UE-2476

Régimen aplicable a los productos agrícolas y a las mercancías resultantes de su transformación originarios de los Estados de África, del Caribe y del Pacífico (ACP), UE-2514

Régimen de ayuda a la exportación de productos del sector de la carne de vacuno que se beneficien de un trato especial a la importación en un tercer país, UE-2494

Régimen de certificados de importación y de exportación en el sector de los cereales y del arroz, UE-2516

Régimen de concesión de restituciones a la exportación para determinados productos agrícolas, UE-2490

Régimen de importación de la carne de porcino, UE-2487

Régimen de importación de leche y productos lácteos y a la apertura de contingentes arancelarios, UE-2527

Régimen de importación y exportación en el sector de la carne de vacuno, UE-2505

Régimen electoral General: EST-66

Régimen especial de estímulo del desarrollo sostenible y la gobernanza a la República de El Salvador, UE-2475

Régimen jurídico de la contabilidad y la información financiera en el Sistema Europeo de Bancos Centrales, UE-2621

Regímenes de ayuda directa en el marco de la política agrícola común, UE-2522, UE-2526 
Regímenes de compras de intervención pública en el sector de la carne de vacuno, UE-2496

Registro Andaluz de Centros de Educación Ambiental: AND-51

Registro comunitario de la flota pesquera, UE-2552

Registro de los contratos y a las comunicaciones de datos en el sector del lúpulo, UE-2488

Registro y transmisión electrónicos de las actividades pesqueras y sobre los medios de teledetección, UE-2559

Registro, evaluación, autorización y restricción de las sustancias y preparados químicos (REACH), UE-2665, UE-2679

Reglamento de Procedimiento del Tribunal de Justicia, UE-2455

Reglamento de Procedimiento del Tribunal de Primera Instancia, UE-2456

Reglamento financiero aplicable al presupuesto general de las Comunidades Europeas, UE-2457

Reglamento interno del Consejo, UE-2454

Regulación del uso de aviones, UE-2586

Requisito de prescripción veterinaria para determinados medicamentos veterinarios destinados a animales productores de alimentos, UE-2534

Responsabilidad medioambiental: EST-97

Restitución a la exportación de determinados productos pertenecientes a los sectores de los huevos y de la carne de aves de corral, UE-2495

Restitución particular por exportación para las carnes deshuesadas de bovinos pesados machos incluidas en el régimen de depósito aduanero antes de la exportación, UE-2502

Restituciones a la exportación en el caso de determinados productos agrícolas, supresión de la prefinanciación, UE-2500

Restituciones adaptadas para los cereales exportados en forma de determinadas bebidas espirituosas, UE-2497

Restituciones por exportación para determinadas conservas de carne de vacuno, UE-2501

Retribución de la actividad de producción de energía electrica: EST-81

Sector del plátano, UE-2525

Seguimiento de los bosques y de las interacciones medioambientales en la Comunidad (Forest Focus), UE-2692

Segundo programa Marco Polo para mejorar el comportamiento medioambiental del sistema de transporte de mercancías (Marco Polo II), UE-2578

Seguridad Social: EST-103

Seguridad Vial: EST-104

Selección de las explotaciones contables para el registro de las rentas en las explotaciones agrícolas, UE-2512 
Séptimo Programa Marco de la Comunidad Europea de la Energía Atómica, UE-2649, UE-2650, UE-2651, UE-2652, UE-2727, UE-2728, UE-2729, UE-2735, UE-2736

Séptimo Programa Marco, UE-2726, UE-2730, UE-2731, UE-2732, UE-2733, UE-2734, UE-2737

Sequia: EST-96

Servicios de atención primaria de salud: AND-54

Servicios en el mercado interior, UE-2677

Simplificación del intercambio de información e inteligencia entre los servicios de seguridad de los Estados miembros de la Unión Europea, UE-2784

Sistema común de tarificación de los servicios de navegación aérea, UE-2577

Sistema común del impuesto sobre el valor añadido, UE-2610, UE-2611

Sistema de acumulación paneuromediterránea del origen, UE-2466

Sistema de certificación del proceso de Kimberley para el comercio internacional de diamantes en bruto, UE-2467

Sistema de Información de Schengen de segunda generación (SIS II), UE-2777, UE-2778, UE-2779, UE-2791

Sistema de Información de Visados, UE-2786

Sistema Financiero: EST-74

Sistemas de calefacción de los vehículos de motor y de sus remolques, UE-2669

Sociedad de la Información: EST-79

Subvenciones: GAL-23

Tasas y Precios Públicos: PV-9

Tipo reducido de IVA, UE-2612

Tormentas de lluvia y viento: EST-71

Transmisión de datos estadísticos sobre los desembarques de productos de la pesca en los Estados miembros, UE-2558, UE-2711

Transparencia de las relaciones financieras entre los Estados miembros y las empresas públicas, UE-2604

Transparencia financiera de determinadas empresas, UE-2604

Transporte de mercancías peligrosas por carretera, UE-2584

Transporte de mercancías peligrosas por ferrocarril, UE-2585

Tratado constitutivo de la Comunidad Europea, versión consolidada, UE-2453

Tratado de la Unión Europea, versión consolidada, UE-2453

Universidades: AND-55; AND-56

Uso de agentes de transformación, UE-2693

Utilización de las tierras retiradas de la producción con vistas a la obtención de materias primas, UE-2499 
Venta de cereales en poder de los organismos de intervención, UE-2477

Vigilancia y al control de los traslados de residuos radiactivos y combustible nuclear gastado, UE-2648

Violencia de género: GAL-24

Volúmenes que activan la imposición de derechos adicionales a las peras, los limones, las manzanas y los calabacines, UE-2509

Volúmenes que activan la imposición de derechos adicionales a los pepinos, las alcachofas, las clementinas, las mandarinas y las naranjas, UE-2492 